\title{
Virtual Reality Application Based on Inertia
}

\author{
Boikhutso Simon Modibane \\ Department of Computer Systems \\ Engineering \\ Tshwane University of Technology \\ Pretoria, South Africa \\ boikhutsomodibane@gmail.com
}

\author{
Pius A Owolawi \\ Department of Computer Systems \\ Engineering \\ Tshwane University of Technology \\ Pretoria, South Africa \\ owolawipa@tut.ac.za
}

\author{
Temitope Mapayi \\ Department of Computer Systems \\ Engineering \\ Tshwane University of Technology \\ Pretoria, South Africa \\ tmapayi@yahoo.com
}

\author{
Vusi Malele \\ Department of Computer Systems \\ Engineering \\ Tshwane University of Technology \\ Pretoria, South Africa \\ vusimalele@gmail.com
}

\author{
Gbolahan Aiyetoro \\ Department of Computer Systems \\ Engineering \\ Tshwane University of Technology \\ Pretoria, South Africa \\ g.aiyetoro@ieee.org
}

Keywords: Virtual Reality, Augmented Reality, 3D Visualization, Computer Graphics, Scientific concept

\section{ACM Reference Format:}

Boikhutso Simon Modibane, Pius A Owolawi, Temitope Mapayi, Vusi Malele, and Gbolahan Aiyetoro. 2021. Virtual Reality Application Based on Inertia. In International Conference on Artificial Intelligence and its Applications (icARTi '21), December 9-10, 2021, Virtual Event, Mauritius. ACM, New York, NY, USA, 7 pages. https://doi.org/10.1145/3487923.3487931

\section{Introduction}

Multiple industries have recognized the promise of immersive Virtual Reality (VR) technology as a significant technological breakthrough for promoting tele-operation scenarios. One of its distinguishing features is the ability to enable operators to imagine abstract concepts, visit locations and interact with objects, or events, that are difficult or impossible to access, due to distance, time, size, or safety concerns. Furthermore, as opposed to the actual existence of people in dangerous situations, VR experiences have safety benefits. The same method is used in education and fully immersive training, where all possible scenarios can be viewed in a VR environment [6]. Researchers believe that VR holds promise for simulation-based training, because the interface retains both the visual-spatial characteristics of the simulated environment and the relation between the student's motor behaviour, and the simulated results. As advances are currently being made to incorporate artificial intelligence (AI) with VR for efficient problem-solving purposes through the display of both large-scale and small-scale spatial information in a three-dimensional format, the endless potential that VR will provide to users are seamlessly wide, as the conversion between various forms of visual modalities and other functionalities will be enhanced [18]. However, the use of VR in the area of learning and development has been defined in a variety of ways. Researchers looked at the use of simulation methods in formal education, and found that there were still 
a number of problems with successful storytelling and determining the effects on student learning outcomes. In training cases, some studies have suggested that the reproduction of scenarios that would be difficult to recreate in real life, is the most successful end use of these encounters. However, all of those reports focused on computer simulations that did not use immersive VR environments, owing to the fact that there were few immersive VR simulations available at the time. Under the circumstances, VR appears to be a viable option. With the introduction of readily available consumer-grade devices, many of the previously mentioned areas have begun to incorporate VR into their training programs [4].

The proposed educational software is being designed to assist learners and teachers in getting a more complete and interesting view of the scientific concept being addressed by it. In this case, the scientific concept of inertia, where we would use a fully interactive, 360-degree artificial world, which will be viewed through a Head Mounted Display (HMD), and the end users can easily interact with it. It also serves to make the topic more engaging for learners, which makes learning easier. The application aims to increase student participation and make learning more engaging. It is also possible to test students' learning on a distance modality using the software created, making it easier for students to go through the simulation as many times as they want, to understand the idea being presented, and to feel in control of an immersive learning process.

\section{Related Works}

A few approaches have been made with respect to the utilization of VR technology over numerous divisions, and businesses have been examined within the writing. The immersive VR in nursing application with a HMD may be a health science application based on nursing instruction, as it implies moving learning forward and increasing understudy inspiration towards nursing and health science [3]. The essential objective of this application is to assist teachers that are battling to discover adequate teaching strategies, mostly due to an increase of security necessities and stiffer monetary imperatives. Constrained to counter a failure of nursing disciples to focus on numerous assignments and "significant execution anxiety", due to lack of customary contact with patients [3]. In any case, there are downsides to the application. Up to now, a HMD in instruction is still used occasionally. This can be due to the reality that in the past, such gadgets were costly and frequently actuate nausea due to low resolution and frame rate, which causes a mismatch between the movement of the head and the corresponding change within the scene [3].

This application is based on a study that utilizes VR to attract youthful and upcoming talent to the field of manufacturing, using inventive presentation of a learning methodology in secondary schools [20]. To do so, the labour-intensive assembling task in a CAVE to introduce young understudies to the concepts of manufacturing is actualized by using an established method that is recreated through an immersive VR [20]. The approach combines game-based learning with VR simulations in interactive environments to enhance learning. The simulation offers a reasonable atmosphere in which the students are able to experiment and discover, enabling them to create their own conceptual model of the setting. Furthermore, the in-built interactive nature of the simulations allows students to see instantaneous outcomes when they develop new models, or examine existing principles based on the modelled ideas [20]. Despite the theoretical and empirical evidence for the potential of the games, the educators still have reservations regarding the general acceptance and usage in learning circles; one explanation for this may be that applications that involve games, which are related to educational activities, are usually dark boxes that are not useful to educators [20]. Another factor, from a learning standpoint, is that games teach common sense skills such as teamwork and problem solving, while the existing system of education focuses on a more precise framework of learning benefits [20].

The work in [17] presents a medical training system outlined to prepare therapeutic practitioners' hand-eye coordination when performing endotracheal intubations. This simulation finishes this assignment with the assistance of VR paradigms [17]. An expansion of this system to medical simulations by utilizing deformable medical models is conceivable. The shared state support of the collaborative AR environment is guaranteed through a novel adaptive synchronization calculation (ASA) that increases the sense of nearness among members, and encourages their interactivity despite infrastructure delays [17]. The system will permit paramedics, and hospital staff to obtain relevant skills and be able to enhance them using VR [17].

Using a system with a combination of virtual people, VR has been applied to construct a virtual fire situation, to illustrate how an evacuation may be conducted in a public sector. The objective of this system is to explore the potential that VR has on emergency management training [10]. The evacuation process has been implemented based on different sources of research and hypothesis in points such as virtual humans, crowd simulation, mental practices beneath weight circumstances, emergency training [10]. Through evaluation results, VR with virtual humans has appeared that they have a potential for utilizing in emergency management training. The virtual space and virtual humans play a critical part in simulating members to feel present and drench amid the training process [10]. The modern side of virtual human conduct and their appearance is restricted to a degree in such simulations, which leads to the participants, who have encounters with online diversions, having a negative perception of the graphical execution amid the virtual evacuation [10]. 
This paper suggests an alternative method of creating a surgical AR system by using commercially available image driven surgery equipment (IGS). It's a prototype for an Augmented Reality (AR) program that gets all the information it needs from a computer for intraoperative navigation For many years, the use of AR in medical applications has been the subject of intensive study. When designing a medical AR scheme, a variety of daunting tasks must be tackled [12]. These include the import and management of medical datasets and preoperatively generated planning data, patient registration in a global coordinate system, precise monitoring of the camera used in the AR setup, as well as the associated surgical instruments. For AR in medicine, most research systems rely on advanced hardware or algorithms. Implementing such foundation technologies can be costly or time-consuming [12].

Manufacturing product maintenance is one of the most common services in manufacturing, with costs sometimes exceeding $30 \%$ of total operating costs. Manufacturers are turning their focus away from products and into a combined ecosystem of Product-Service Systems (PSS). Hence, the key objective of this project is to develop a service-oriented system that is based in the cloud and utilizes AR technology to ensure collaborative repairs can be done remotely between manufacturers and on-site technicians.[13]. This system provides techniques for the end user to document the failure, the behaviour needed of the expert to provide repair guidance in an AR application, and a cloud-based interface that will enable them to communicate and share information [13]. This system is also comprised of automated smart assembly and disassembly procedures for the generation of AR scenes and assembly chains, as well as an enhanced interface, with the aim of maximizing existing information use while generating vibrant AR service instructions [13]. The developed device has been tested in a real-world scenario based on the needs of a small/ medium enterprise's (SME) automation.

In the manufacturing sector, where the generation of instantaneous reports for the purpose of decision-making are important, AR as an information visualization tool can be used. A company must ensure that its processes are continuously controlled and strengthened. As a result, Computer Aided Quality (CAQ) Software, linked with an AR solution is developed to achieve a production management system. An AR aided solution is used to display Key Performance Indicators (KPI) for every device within an industrial plant. These KPI are obtained from the measuring sensors and incorporated into the CAQ Software. The obtained information is transferred to a mobile device via wireless medium and positioned using an Indoor Positioning System (IPS) [16]. The application of this solution yields a dynamic tool that allows audit times to be cut in half.[16]

The recent increase in popularity of personal mobile devices has resulted in major changes in information search and distribution. The newly developed AR technology, in particular, allowed a ground-breaking method of knowledge acquisition by bringing additional virtual world information into the real world. A service on a mobile application platform that can be used to effectively disseminate information on advertising to end users is introduced and developed using the markerless AR system on smart mobile devices [5]. The service developed in this research work is integrated with the database management and AR systems to rapidly deliver more precise and diverse information to end users, while traditional advertisement applications merely implement and illustrate products by enticing customers to have an interest [5].

One drawing can be worth hundreds of words when sharing details about geometric figures, so visualisation aids developed for complex three-dimensional (3D) solid objects are extremely useful for students and educators. The concept of using the Virtual Reality Modelling Language (VRML) to visualize 3D structures in a networked environment for middle school geometry classes is explained in this paper. The paper also demonstrates how useful it is for both teachers and students [19]. The VRML objects obtained from the server are used by the educators in class. The students can study and discover more about these objects by connecting to the educators' server through their internet access [19]. The use of 3D objects that are designed using VRML has a meaningful effect on the learning of the students regarding subjects that are related to geometric. This is verified in the test results that shows the comparison between normal classes that only depend on oral explanation or using pencil and paper, and the geometry classes designed using VRML [19].

The advancement of VR technology has had a major impact on a variety of sectors, including medicine. This paper briefly discusses the use of VR from two perspectives. Digital anatomy and virtual human projects have been extensively studied in numerous universities and institutions around the world and can provide medical students with a more practical visual sense than conventional teaching approaches based on 2-D diagrams [7]. And the VR surgical system, which provides surgeons with a simulated environment with vibrant visual, auditory, and haptic senses, would be an excellent replacement for cadavers, because residents and interns will repeat all kinds of operations in the system without limitations [7].

Landmines are often used as both a defence and an assault weapon. To address the drawbacks previously outlined, the research is focused on developing a landmine detection training simulation, which supports real-world landscape conditions and provides a secure environment that can be used to detect the landmines. The simulation software that was developed in this study is focused on VR technology. A Detector Appearance Control (DAC) system controller designed specifically for this simulator, as well as the Kinect controller, provide interaction with the computer [1]. When 
compared to current landmine detection instruction, the simulator offers significant time and cost savings. Simultaneously, detections were carried out in a secure environment free of risk factors, and real-world land conditions are provided. In addition, the DAC system controller, such as the Kinect system, can accurately detect the exact space coordinates of the joints in the human body [1].

In this paper, a VR surgical simulator is created, and the device successfully simulates the key procedures of phacoemulsification cataract surgery [8]. The training system's simulation results show that the built simulator can create a simulated environment where surgery procedures can be carried out with accurate force feedback for postgraduate medical trainees, to perform their training exercises using a computer and a pair of force-feedback haptic devices [8]. Furthermore, the effective simulation of mesh modifications on the human eyeball, with visual realism and consistent force feedback during the surgical procedure, demonstrates that the simulator that was developed can be used to train surgeons' surgical skills [8].

Researchers and practitioners will now introduce stereoscopic virtual reality (StereoVR) to consumers' homes due to the success of home-based stereoscopic television. This study is centred on comprehending the shopping experiences of consumers in this novel medium to study the future advancement of using StereoVR in the retail market sector [9]. StereoVR, according to this study, has the potential to become a new area for the interactive industry. To investigate the possible applications of this technology in the retail market sector, the authors developed and created a StereoVR called "FutureShop," which can be used to introduce virtual fashion retailing practices and gather customer feedback for future growth [9]. The purchasing intention of customers, digital shopping, and hedonic shopping experience were all factors investigated in this study. The results and implications indicate that by improving consumers' hedonic shopping experiences in the StereoVR, the StereoVR will make a major contribution to developing more immersive experiences for apparel retailing [9].

The use e of VR in the e-commerce sector has a big potential for translating online shopping into a physical shopping experience. However, an increasing body of research focuses on the use of VR technologies in the creation of e-commerce environments, with little or no attention to the social and behavioural aspects of online shopping, for instance, confidence. No doubt, confidence is one of the key success indicators for e-commerce, though, how it will be achieved in an online store remains a mystery. The use of VR in online shopping environments has been demonstrated to provide a superior user experience compared to traditional online stores, in this paper. This also allows for the development of confidence for the customer [14]. Furthermore, a virtual shopping mall prototype, based on concepts drawn from an empirically validated model designed for the improvement of e-commerce confidence, is introduced [14].

This paper offers a summary of VR's applications in the educational sector. It focuses on three different school projects [15]. There is a distinct connection between the learning environment and the virtual world experience with both ventures. The focus here would be on the broader contribution that these initiatives can bring to our understanding of virtual worlds, rather than on their pedagogical importance, which has been recorded elsewhere [15]. To do so, it looks at how VR technologies are incorporated into the syllabus, how they contribute to the learning and teaching processes, the systems' accessibility, and the virtual worlds' possibilities and constraints in each case [15]. When compared to other evolving VR technologies, it can be observed that various systems provide a range of options for creating presence in virtual worlds and communicating with them.

3D worlds are becoming more open over the Internet as personal computer power and usable bandwidths increase, enabling audiences to easily discover virtual built worlds. Even with advancements in bandwidth and computational power, developing practical 3D environments that are quick to download, and enjoyable to walk around, necessitate the use of a variety of modelling methods. The explanation of the design methods used in the remodelling of a web-based VR recreation of a historical site is presented in this paper. It is worth noting that there was much emphasis in the paper on various methods that can be used to reduce the duration of download while maintaining historical realism [2]. Any designer can use the techniques and processes as a guide to help create lightweight functional virtual models. The research and development of a prototype dental simulator for the procedural training was proposed in [11]. The simulator utilizes VR and haptics technologies to teach trainees how to perform periodontal disease diagnosis and treatment by visualizing a 3-dimensional graphical image of the human mouth and experiencing actual tactile feelings by moving the exterior part of teeth, and other parts of it using the dental instruments virtually incorporated [11]. The haptics utilized in the procedure are undeniably necessary for an accurate usage of the simulator, because periodontics allows dentists to rely primarily on tactile stimuli to perform medical diagnostic processes [11]. An experiment performed by the University of Illinois at Chicago (UIC) validated the VR training simulator, demonstrating the simulator's technical contribution and usefulness as a critical part of the Department of Periodontics' curriculum [11].

\section{Methodology}

In this section, the methodology description and the tools used in this study are presented. 


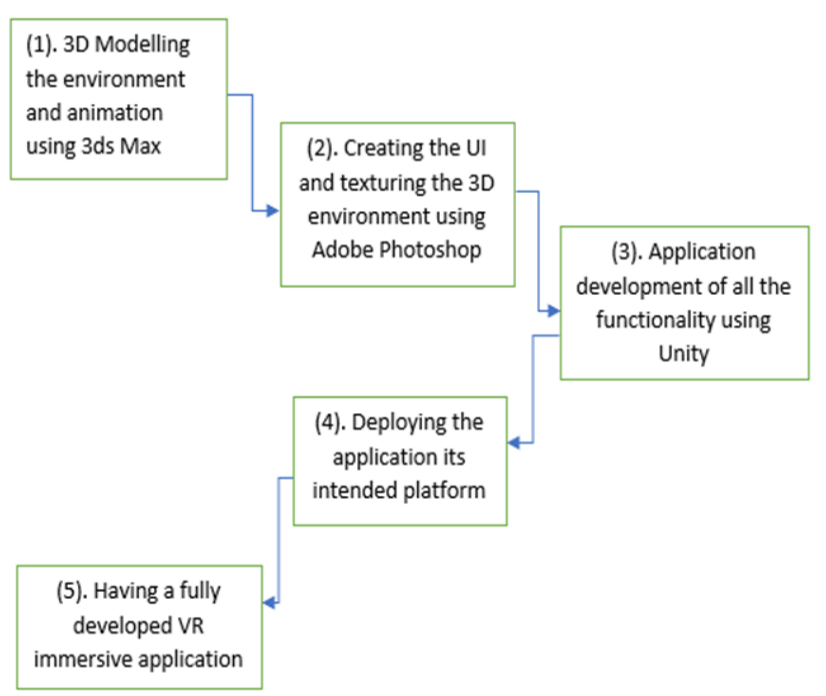

Figure 1. Diagrammatic description of the system.

\subsection{Methodology Description}

The block diagram of the proposed VR educational scientific application, based on the concept on inertia, is shown in Figure 1 and it explains the process of each step that was taken during the development of this application.

The process starts by developing all the 3D models that will be required, including the $3 \mathrm{D}$ environment that the application will be based on, followed by the animation that was used in the development process, and will be done using the 3ds Max software. With the use of Adobe Photoshop, the 3D environment and 3D models were textured and given color to get everything to look and feel as realistic as possible. The same software was used to make all the user interface that the user will be interacting with to perform certain actions within the application. The final step was to then use the Unity software to add and develop all the functionalities that are within the applications, and test and debug every functionality to make sure that it does what it is intended to do.

\subsection{Elements/Components Description}

In this section, we will go over the different components that were used for the entire system. Since this will be a VR application, the majority of what will be explained is going to be the software used, and only a few hardware related components used in the system.

Autodesk 3ds Max. Autodesk 3ds Max is a 3D graphics software skillfully used for the creation of 3D images and animations with different models for various games. Its prior names were 3D Studio with 3D Studio Max.

Unity. Unity Technologies is a company that makes a crossplatform game engine. The engine can be used to render games in three dimensions, two dimensions, VR, and AR, as well as simulations and other experiences.

Microsoft (MS) Visual Studio. MS Visual Studio is a Microsoft integrated development environment. It is utilized for the rendering of blogs, web applications and services with mobile applications on smartphones.

Adobe Photoshop. Adobe Photoshop was released by Adobe Inc and used for the editing of graphics of different operating systems. Photoshop makes provisions for masks, alpha compositing, and many color templates, including RGB, spot color, and duotone, and can be used for the editing and composition of raster images in manifold layers.

Head Mounted Display. Smartphones are used to have a VR environment with Head Mounted Display. Users simply insert their smartphones into the headset, and the device will appear directly in front of their eyes, with a series of lenses that provide a sense of depth. The quality of the VR experience is, in fact, determined by the smartphone used. The form of screen and its resolution are two variables to consider.

Smartphone. We have selected an android smartphone as the platform that we will be deploying, and we will use it to show the functionalities of application and capabilities of the VR technology. This is a list of the minimum specifications that are needed on any android smartphone for it to support VR applications:

Resolution: It is recommended that we use a smartphone with a resolution not lower than Full HD, which is $1920 \mathrm{x}$ 1080 pixels.

Gyroscope: The existence of a gyroscope is needed to rotate the head and look around in VR, since the gyroscope decides the device's orientation in three-dimensional space.

Accelerometer: In mobile phones, accelerometers are used to detect the phone's orientation and calculate linear acceleration of movement.

$R A M$ : You will also require minimum of 2 GB RAM to process all the functionalities of the application at a reasonable speed.

\subsection{Algorithm Description}

The algorithm used for the VR system designed in this study is presented using the flowchart described in this subsection. 


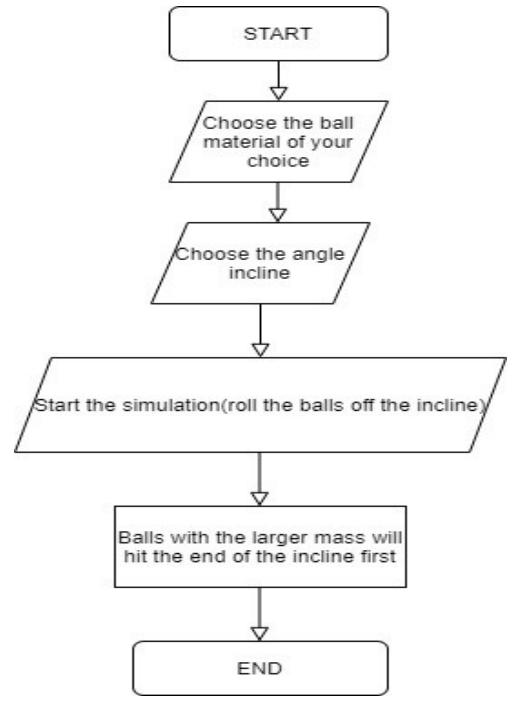

Figure 2. Flowchart description of the designed system

The flowchart diagram in Figure 2 describes the main function of the simulation, where the concept of inertia will be illustrated. The more mass an object has, the more inertia it will have, and using this notion, we were able to make use of objects with different masses, on a slope with different include angles, and from that we were able to prove the concept derived from the notion mentioned above.

\section{Results and Discussion}

The diagrams in Figure 3 and Figure 4 show the proposed VR application. Figure 3 shows the user interface that the user will be interacting with, while it switches from one simulation scene to the next, and Figure 4 shows the 3D environment that the simulation/experiment will take place in, with all the 3D objects necessary to perform the simulation.

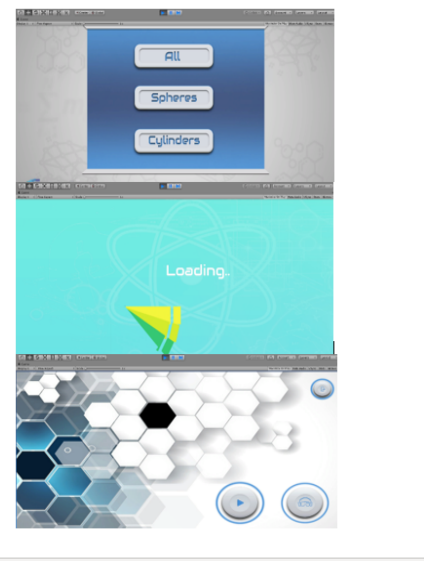

Figure 3. User Interface of the proposed system
Table 1. The comparison between traditional teaching methods and VR simulations.

\begin{tabular}{|l|l|l|}
\hline & \multicolumn{2}{|c|}{ Marks (In Percentage \%) } \\
\hline Users & $\begin{array}{l}\text { Traditional Teaching } \\
\text { Method }\end{array}$ & $\begin{array}{l}\text { Virtual Reality Simu- } \\
\text { lations }\end{array}$ \\
\hline User 1 & 55 & 70 \\
\hline User 2 & 36 & 63 \\
\hline User 3 & 75 & 90 \\
\hline User 4 & 48 & 69 \\
\hline User 5 & 65 & 80 \\
\hline User 6 & 75 & 70 \\
\hline User 7 & 47 & 69 \\
\hline User 8 & 69 & 64 \\
\hline
\end{tabular}

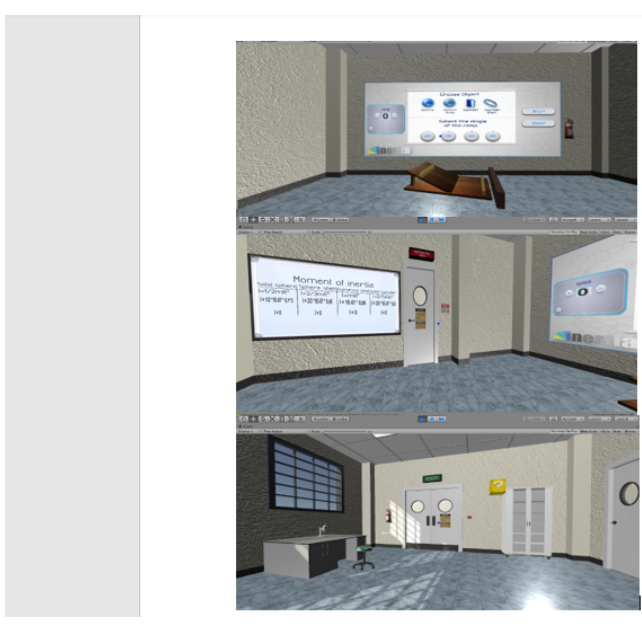

Figure 4. 3D Environment

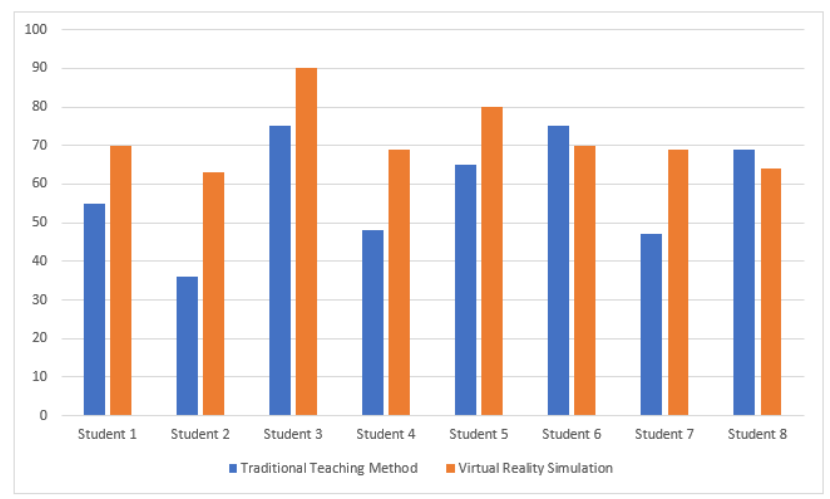

Figure 5. Performance comparison of the Proposed VR Application

Table 1 and Figure 5 present the results of users' obtained from this proposed system. We compared scores of the users taught the concept of inertia using the traditional teaching 
method, and those that were exposed to the VR application based on the same concept of inertia, which is the proposed simulation that is presented in this paper. The results show that the users performed much better when they were exposed to the concept using the VR application other than the normal traditional way of teaching by approximately 15 to 27 per cent.

\section{Conclusion}

This study represents a virtual reality application based on the concept of inertia, and this was done by using the combination of 3D visualization tools such as 3Ds Max and Unity 3D software deployed for mobile. The study also presented a positive outcome, with the results showing a significant change in the studies of the learners after they were exposed to the system, proving that the use of system does improve the ability of the learners to comprehend a certain concept or experiment. In future, we shall implement adding more scientific concepts and experiments, so the application/system can serve as a fully flashed science lab for unprivileged learners with no access to this, so that they can have equal access to quality education as do their counterparts.

\section{References}

[1] Merve Varol Arısoy and Ecir Uğur Küçüksille. 2021. Landmine detection training simulation using virtual reality technology. Virtual Reality 25, 2 (2021), 461-490.

[2] Kevin Badni. 2006. Virtual reality design techniques for web-based historical reconstructions. Virtual Reality 9, 4 (2006), 215-225.

[3] Liz Mary Ditzel, Karole Hogarth, and Raewyn Lesa. 2017. Immersive learning in nursing education: Results of a study. fournal of Nursing Education and Practice 7, 5 (2017), 120-130.

[4] James Elliman, Michael Loizou, and Fernando Loizides. 2016. Virtual reality simulation training for student nurse education. In 2016 8th International Conference on Games and Virtual Worlds for Serious Applications (VS-Games). IEEE, 1-2.

[5] Young-geun Kim and Won-jung Kim. 2014. Implementation of augmented reality system for smartphone advertisements. international journal of multimedia and ubiquitous engineering 9, 2 (2014), 385-392.

[6] Marios Kyriakou, Xueni Pan, and Yiorgos Chrysanthou. 2017. Interaction with virtual crowd in Immersive and semi-Immersive Virtual Reality systems. Computer Animation and Virtual Worlds 28, 5 (2017), e1729.

[7] Pinhua Lai and Wenjian Zou. 2018. The application of virtual reality technology in medical education and training. Global fournal of Information Technology: Emerging Technologies 8, 1 (2018), 10-15.

[8] Chee Kiang Lam, Kenneth Sundaraj, and M Nazri Sulaiman. 2014 Computer-based virtual reality simulator for phacoemulsification cataract surgery training. Virtual Reality 18, 4 (2014), 281-293.

[9] Kung Wong Lau and Pui Yuen Lee. 2019. Shopping in virtual reality: a study on consumers' shopping experience in a stereoscopic virtual reality. Virtual Reality 23, 3 (2019), 255-268.

[10] Lei Li, Maojun Zhang, Fangjiang Xu, and Shaohua Liu. 2005. ERT-VR: an immersive virtual reality system for emergency rescue training. Virtual reality 8, 3 (2005), 194-197.

[11] Cristian Luciano, Pat Banerjee, and Thomas DeFanti. 2009. Hapticsbased virtual reality periodontal training simulator. Virtual reality 13 , 2 (2009), 69-85.
[12] R Marmulla, Harald Hoppe, J Mühling, and G Eggers. 2005. An augmented reality system for image-guided surgery: This article is derived from a previous article published in the journal International Congress Series. International journal of oral and maxillofacial surgery 34, 6 (2005), 594-596.

[13] Dimitris Mourtzis, Vasilios Zogopoulos, and E Vlachou. 2017. Augmented reality application to support remote maintenance as a service in the robotics industry. Procedia Cirp 63 (2017), 46-51.

[14] Panagiota Papadopoulou. 2007. Applying virtual reality for trustbuilding e-commerce environments. Virtual Reality 11, 2-3 (2007), 107-127.

[15] Ralph Schroeder. 1995. Learning from virtual reality applications in education. Virtual Reality 1, 1 (1995), 33-39.

[16] Daniel Segovia, Miguel Mendoza, Eloy Mendoza, and Eduardo González. 2015. Augmented reality as a tool for production and quality monitoring. Procedia Computer Science 75 (2015), 291-300.

[17] RC Shetty. 2006. Augmented Reality Training Kit Can Offer Novel Teaching Solution For Future Cardiac Surgery And Other Medical Professionals. The Internet fournal of Medical Simulation 2, 1 (2006).

[18] Alexander W Siegel. 1981. The externalization of cognitive maps by children and adults: In search of ways to ask better questions. Spatial representation and behavior across the life span (1981), 167-194.

[19] Ki-Sang Song and Woo Yeol Lee. 2002. A virtual reality application for geometry classes. Journal of Computer Assisted Learning 18, 2 (2002), 149-156.

[20] Antoniou Stratos, Rentzos Loukas, Mavrikios Dimitris, Georgoulias Konstantinos, Mourtzis Dimitris, and Chryssolouris George. 2016. A virtual reality application to attract young talents to manufacturing. Procedia CIRP 57 (2016), 134-139. 